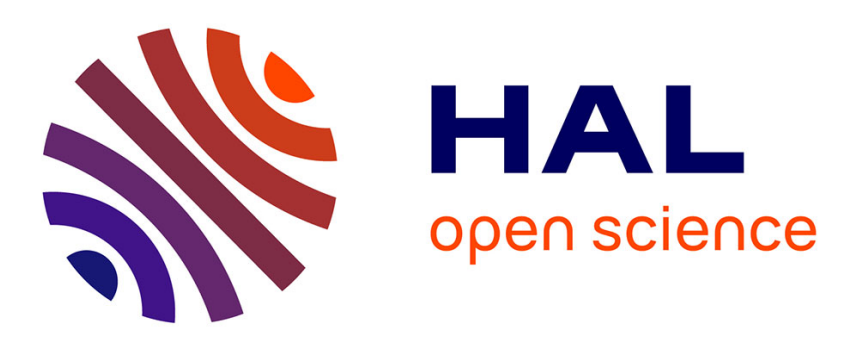

\title{
Mathematical Modelings of Some Smart Materials and Structures
}

\author{
Christian Licht, Thibaut Weller
}

\section{To cite this version:}

Christian Licht, Thibaut Weller. Mathematical Modelings of Some Smart Materials and Structures. Applied Mechanics and Materials, 2015, 749, pp.13-16. 10.4028/www.scientific.net/AMM.749.13 . hal-01367475

\section{HAL Id: hal-01367475 https://hal.science/hal-01367475}

Submitted on 16 Sep 2016

HAL is a multi-disciplinary open access archive for the deposit and dissemination of scientific research documents, whether they are published or not. The documents may come from teaching and research institutions in France or abroad, or from public or private research centers.
L'archive ouverte pluridisciplinaire HAL, est destinée au dépôt et à la diffusion de documents scientifiques de niveau recherche, publiés ou non, émanant des établissements d'enseignement et de recherche français ou étrangers, des laboratoires publics ou privés. 


\title{
Mathematical Modelings of Some Smart Materials and Structures
}

\author{
Christian Licht ${ }^{1, a *}$ and Thibaut Weller ${ }^{1, b}$ \\ ${ }^{1}$ Laboratoire de Mécanique et Génie Civil, UMR 5508 CNRS-Université Montpellier, c.c. 48, Place \\ Eugène Bataillon, 34095 Montpellier cedex 05, France \\ aclicht@univ-montp2.fr, ${ }^{b}$ thibaut.weller@univ-montp2.fr \\ ${ }^{*}$ Corresponding author
}

Keywords: Smart materials, Smart structures, Mathematical modeling.

\begin{abstract}
Models of smart materials and structures are derived through rigorous mathematical methods. We establish a classification of piezoelectric and piezomagnetic crystalline materials and propose simplified but accurate models of thin structures made of piezoelectric or electromagneto-elastic materials.
\end{abstract}

\section{Introduction}

Smart materials present significant multi-physical couplings, one of the fields of interaction being mechanics. For instance, the application of an electric field on a piezoelectric material generates a deformation and conversely, a deformation generates polarization. Piezoelectric materials are widely used in the design of smart structures. They are integrated in these structures, which take technological advantages of the piezoelectric effect. We may distinguish two kinds of devices: sensors and actuators. Sensors transform a mechanical loading into an electric signal and the systems they enter convey these signals according to the needs: design of micro weighing machine, airbags, Hi-Fi equipments... The actuators convert an electric signal into a displacement, which can be monitored with precision, of say mirrors, lenses, machine tools... This twofold behavior is known and used for a long time and several derivations of models of smart structures can be found in the engineering literature, but, due to the various used methods, discrepancies and controversies may occur. Here we aim to present rigorous mathematical models of both smart materials and smart structures by mainly confining to the piezoelectric or electromagneto-elastic cases.

First we establish a classification of piezoelectric and piezomagnetic crystalline materials by a suitable description of crystal lattices and a careful study of the symmetries of the tensors, which account for these two phenomena. We mainly used algebraic tools from representation theory. In a second part, we intend to propose simplified but accurate models of structures made of piezoelectric or elecromagneto-elastic materials, these structures (thin plates, slender rods) presenting one or two small dimensions. The models are obtained by a rigorous study of the asymptotic behavior of a three dimensional body when some of its dimensions, considered as parameters, tend to zero. We used various tools of variational and functional analysis, the point being to consider boundary value problems depending on small parameters. This study has been carried out in the steady state and transient cases.

\section{Modeling of Smart Materials}

Because multi-physical couplings of a material strongly depend on its crystalline structure and may vanish for some crystal classes, it is necessary to find a way to classify the microstructure of such material. It is for example well known that an isotropic material cannot be piezoelectric.

Considering the crystalline state, it can be classified into 32 families called crystal classes. However, only 20 of these crystal classes can give birth to the piezoelectric phenomenon. This discrepancy lies in the fact that crystal symmetries and tensors symmetries are two distinct concepts. Working on these concepts, we were able to show that the 20 piezoelectric crystal classes may in fact 
be classified in 15 equivalence classes. This classification has been obtained thanks to two different kinds of mathematical tools. First, we modeled the crystal structure itself. Second, we studied the symmetry of piezoelectric tensors.

We therefore chose in [1] and [2] to study crystals as geometrical objects in order to rigorously define a suitable classification with respect to their different kinds of symmetries. This paves the way to see crystals as algebraic objects. It is therefore possible to define geometric and arithmetic equivalent classes which enclose all their symmetry properties.

However, the goal is to describe the symmetries of the piezoelectric phenomenon, not only the symmetries of piezoelectric crystals. The symmetries of the piezoelectric phenomenon are of macroscopic nature while the symmetries of piezoelectric crystals are of microscopic nature. To fill this gap, we need energetic arguments. The validity of these arguments are based on the Cauchy-Born hypothesis which states that the position of atoms (which is a microscopic information) is given by the deformation gradient of the solid (which is a macroscopic information). Another very important tool in this change of scale between microscopic and macroscopic informations is the Pitteri-Ericksen Theorem which allows to study the deformation of the solid in a reduced domain called Pitteri-Ericksen neighborhood. This kind of neighborhood has the fundamental property that the symmetry properties are finite groups.

These two steps are necessary to define the right actions to consider for the study and classification of tensors symmetries. To make it short, the method is to « rotate » tensors and to observe when these « rotations » let them unchanged. Two different kinds of tools make this manipulations easier: the harmonic and Cartan decompositions. The goal of these decompositions is to associate a polynomial to a given tensor. The advantage of polynomials is that they are much easier to " rotate $»$ ! It is then possible to show that piezoelectric tensors symmetries can be classified in 15 equivalent classes.

We also produced an extension of this method to piezomagnetism in [3]. The specificity of this phenomenon is that we need to consider spatio-temporal symmetries. The description is therefore richer in the case of piezomagnetism.

\section{Modeling of Smart Structures}

First, finding the equilibrium configuration of a linearly piezoelectric plate can be formulated in terms of a linear elliptic boundary value problem posed in a cylindrical domain $\omega \times(-\varepsilon, \varepsilon)$, with $\omega$ being a two-dimensional domain. To get a simplified but accurate enough model, we take advantage of the small thickness $2 \varepsilon$ of the plate by studying the asymptotic behavior of the displacement and electrical potential fields when $\varepsilon$ goes to zero. By some techniques of singular perturbation in variational formulations, [4] establishes that this asymptotic behavior is essentially described by a linear elliptic boundary value problem posed in the sole domain $\omega$. It can be shown that, depending on the type of electrical loading, two kinds of limit generalized kinematics occur with different numbers of degrees of freedom. Moreover, in the case of a polarization normal to the plate, we investigate the influence of crystalline symmetries on the properties of our models and show that some crystal classes lead to a striking structural switch-off of the piezoelectric effect: even if the material is piezoelectric, it is not anymore the case for the thin plate. More precisely, this switch-off does not depend only on the crystal class of the piezoelectric material that constitutes the plate but also on the electrical boundary conditions, i.e. crystal symmetries do not have the same influence on sensors and actuators. It is shown that if the plate is used as a sensor, the decoupling occurs for the classes $2 ; 222 ; 2 \mathrm{~mm} ; 4 ; \overline{4} ; 422 ; 4 \mathrm{~mm}, \overline{4} 2 \mathrm{~m} ; 6 ; 622 ; 6 \mathrm{~mm} ; 23$ and $\overline{4} 3 \mathrm{~m}$. While, if the plate is used as an actuator, the decoupling takes place with the classes $m ; 32 ; 422 ; \overline{6} ; 622$ and $\overline{6} m 2$. We therefore observe that the decoupling occurs for sensors and actuators for only two classes: 422 and 622 !

Besides the piezoelectric coupling, some materials are sensitive to magnetic effects, thus in [5] we extended the previous modeling to linearly electromagneto-elastic thin plates. A similar mathematical analysis of the asymptotic behavior of the displacement, electrical and magnetic potentials can be done to derive new models. The novelty here is that four limit behaviors may appear 
according to the type of boundary conditions and the magnitude of the data on the electric and magnetic fields. These cases can be described as previously but by a couple of indices $(p, q) \in\{1,2\}^{2}$ in place of a sole index $p$. The physical situation when the thin plate is used as an electrical (resp. magnetic) sensor corresponds to $p=1$ (resp. $q=1$ ) while the actuator case corresponds to $p, q=2$. It therefore appears two original mixed behaviors when $p \neq q$. In these situations, the plate is at the same time a sensor and an actuator excepted for the classes for which the plate is no more electromagneto-elastic. The two cases $p \neq q$ allow the modeling of electrically commanded magnetic devices and of magnetically commanded electric ones, which is of considerable interest in the development of non-volatile magnetic random access memories.

From a technological point of view, piezoelectric materials can also be used in wires or slender rods. Now, the reference configuration of the piezoelectric structure is $\varepsilon \omega \times(0, L)$, with $\mathrm{L}$ a fixed positive real number. On the contrary to the case of plates, the state variables of the limit model obtained in [6] do not reduce to the couple displacement/electrical potential but involve additional variables: two fields of displacements (easy to interpret mechanically) and a scalar field of electrical nature. Nevertheless the kinematics of the state variables is simpler than the one of the genuine three-dimensional model which is very favourable from a numerical point of view. As in the purely elastic case it is worthwhile to note that for particular classes of monoclinic materials the additional variables disappear. Anyway, in the sensor case, the additional variables can be eliminated but it leads to non standard equations involving non local terms!

The interest of an efficient modeling of the dynamic response of piezoelectric plates lies in the fact that a major technological application of piezoelectric effects is the control of vibrations of structures through very thin plates or patches. We proposed two modelings [4,7] depending on the various extents to which the magnetic effects are taken into account. Actually, because of the large discrepancy between the celerities of the mechanical and electromechanical waves, magnetic effects can be disregarded. That is why we propose a modeling [4] in the appropriate framework of the quasi-electrostatic approximation which claims that the electrical field still derives from an electrical potential. In the two cases, we still obtained two kinds of models (sensor or actuator) and showed that the limit constitutive equations obtained in the static asymptotic models remains valuable in the dynamic case.

As the Mindlin's theory of elastic dielectrics with polarization gradient accommodates the observed and experimentally measured phenomena, such as electromechanical interactions in centrosymmetric materials, capacitance of thin dielectric films, surface energy of polarization, deformation and optical activity in quartz, we performed [8] a mathematical modeling of the second order piezoelectric thin plate. Depending on the type of electric loading, three different models appear at the limit. For two of them and for any symmetry class, the constitutive laws can be expressed as a Schur complement of the second order piezoelectric tensor. It seems very likely that non-local terms appear in the remaining case as for slender rods. Moreover we show that, even for the second order piezoelectricity, an electromechanical switch-off may appear in the structure if the plate is designed with specific materials.

As the essential technological interest of piezoelectric patches is the monitoring of a deformable body they are bonded to, we proposed [9] various asymptotic models for the behavior of the body through the study of the system constituted by a very thin linearly piezoelectric flat patch perfectly bonded to a linearly elastic three-dimensional body. We obtained four limit models corresponding to reinforcement of the body along the bonded surface. Two of these models are purely elastic reinforcements while the two others imply sensing or actuating behaviors.

\section{Concluding Remarks}

The main advantage of the reduction dimension process is that it allows easy numerical experiments by avoiding the meshing of thin three-dimensional domains. 
The methods presented here can be extended to other kinds of multi-physical couplings such as the one arising in quasicrystalline media (see [10]).

\section{References}

[1] T. Weller, Etude des symétries et modèles de plaques en piézoélectricité linéarisé, Thèse, Université Montpellier 2, 2004.

[2] G. Geymonat, T. Weller, Classes de symétrie des solides piézoélectriques, Comptes Rendus Mathématiques, 335 (2002) 847-852.

[3] T. Weller, G. Geymonat, Piezomagnetic tensors symmetries: an unifying tentative approach, in: V.K. Kalpakides, G.A. Maugin (Eds.), Configurational Mechanics, A.A. Balkema Publishers, 2004, pp. 87-105.

[4] C. Licht, T. Weller, Asymptotic modeling of thin piezoelectric plates, Annals of Solid and Structural Mechanics, 1 (2010) 173-188.

[5] T. Weller, C. Licht, Modeling of linearly electromagnetoelastic thin plates, C. R. Mécanique 335 (2007) 201-206.

[6] T. Weller, C. Licht, Asymptotic Modeling of linearly piezoelectric slender rods, C. R. Mécanique 336 (2008) 572-577.

[7] C. Licht, Asymptotic Modeling of Thin Linearly Piezoelectric Plates taking into account Dynamical Electro-Magnetic Effects, International Conference on Mathematical Analysis and Its Applications ICMAA 2006, Bangkok, Thailand, 20-24 May 2006.

[8] T. Weller, C. Licht, Asymptotic Modeling of piezoelectric plates with electric field gradient, C. R. Mécanique 340 (2012) 405-410.

[9] C. Licht, S. Orankitjaroen, P. Viriyasrisuwattana, T. Weller, Bonding a linearly piezoelectric patch on a linearly elastic body, C. R. Mécanique 342 (2014) 234-239.

[10] T. Weller, C. Licht, Asymptotic modeling of thin linearly quasicrystalline plates, C. R. Mécanique 341 (2013) 793-798. 